\title{
вмј Global Health Digital adherence technologies for the management of tuberculosis therapy: mapping the landscape and research priorities
}

\author{
Ramnath Subbaraman, ${ }^{1,2}$ Laura de Mondesert, ${ }^{3}$ Angella Musiimenta, ${ }^{4}$ \\ Madhukar Pai, ${ }^{5}$ Kenneth H Mayer, ${ }^{6,7}$ Beena E Thomas, ${ }^{8}$ Jessica Haberer ${ }^{9}$
}

To cite: Subbaraman $\mathrm{R}$, de Mondesert L, Musiimenta A, et al. Digital adherence technologies for the management of tuberculosis therapy: mapping the landscape and research priorities. BMJ Glob Health 2018;3:e001018. doi:10.1136/ bmjgh-2018-001018

Handling editor Seye Abimbola

- Additional material is published online only. To view please visit the journal online (http://dx.doi.org/10.1136/ bmjgh-2018-001018).

Received 21 June 2018 Revised 13 September 2018 Accepted 14 September 2018

A Check for updates

(c) Author(s) (or their employer(s)) 2018. Re-use permitted under CC BY. Published by BMJ.

For numbered affiliations see end of article.

Correspondence to Dr Ramnath Subbaraman; ramnath.sub@gmail.com

\section{ABSTRACT}

Poor medication adherence may increase rates of loss to follow-up, disease relapse and drug resistance for individuals with active tuberculosis (TB). While TB programmes have historically used directly observed therapy (DOT) to address adherence, concerns have been raised about the patient burden, ethical limitations, effectiveness in improving treatment outcomes and long-term feasibility of DOT for health systems. Digital adherence technologies (DATs) — which include feature phone-based and smartphone-based technologies, digital pillboxes and ingestible sensors-may facilitate more patient-centric approaches for monitoring adherence, though available data are limited. Depending on the specific technology, DATs may help to remind patients to take their medications, facilitate digital observation of pilltaking, compile dosing histories and triage patients based on their level of adherence, which can facilitate provision of individualised care by TB programmes to patients with varied levels of risk. Research is needed to understand whether DATs are acceptable to patients and healthcare providers, accurate for measuring adherence, effective in improving treatment outcomes and impactful in improving health system efficiency. In this article, we describe the landscape of DATs that are being used in research or clinical practice by TB programmes and highlight priorities for research.

\section{INTRODUCTION}

Tuberculosis (TB) is the leading infectious cause of death globally, even though most forms of TB are curable. ${ }^{1}$ The risks of death, disease relapse and acquired drug resistance increase with irregular adherence to $\mathrm{TB}$ therapy. ${ }^{23}$ Compared with drug-sensitive TB, drug-resistant strains require an extended duration of therapy with second-line or thirdline drugs that are less effective, have higher rates of adverse effects and are more expensive.

Causes of medication non-adherence are complex and include psychosocial (eg,
Summary box

- Digital adherence technologies (DATs) — which include feature phone-based and smartphone-based technologies, digital pillboxes and ingestible sensors-have the potential to facilitate more patient-centric approaches for monitoring tuberculosis (TB) medication adherence than existing directly observed therapy (DOT) models.

- DATs may serve a variety of functions in TB care, including reminding patients to take their medications, facilitating digital observation of pill-taking, compiling patient dosing histories and triaging patients based on their level of adherence, which can facilitate provision of individualised ('differentiated') care.

- Evidence that DATs improve TB treatment outcomes is limited, and more robust research is needed to understand the acceptability, accuracy, clinical effectiveness and cost-effectiveness of these technologies.

- DATs should be integrated with clinical strategies for identifying and addressing the underlying psychosocial, medical, structural and health system-related causes of medication non-adherence; otherwise implementation of DATs may run the risk of overly focusing on 'observation' and replicating paternalistic aspects of existing DOT models.

alcohol use, ${ }^{4}$ depression, ${ }^{5}$ stigma $\left.{ }^{6}\right)$, structural (eg, distance from clinics, medication costs), therapy-related (eg, toxicities ${ }^{5}$ ) and health system-related barriers (eg, lack of counselling, poor user-experience with the health system). ${ }^{78}$ Directly observed therapy (DOT) was designed to reduce non-adherence; however, concerns have been raised that some DOT approaches may impinge on patient autonomy ${ }^{9}$ and have minimal efficacy for improving treatment outcomes, as compared with self-administered therapy. ${ }^{10-12}$

With the expansion of mobile phone and cellular access-including in high-TBburden countries in Africa, ${ }^{13}$ Asia and Latin 
America-digital adherence technologies (DATs) may facilitate alternative approaches for improving adherence. These technologies range from cellphone short messaging service (SMS) texts, to digital pillboxes, to ingestible sensors. DATs use cellular communication and other innovations to perform a variety of functions, including reminding patients to take medications, digitally observing doses taken and compiling dosing histories that can be used by healthcare providers (HCPs) to identify and intervene on non-adherence. DATs have been shown to improve adherence in patients with HIV, ${ }^{14-17}$ diabetes $^{18}$ and other conditions. ${ }^{1920}$ Fewer studies have evaluated whether these changes in adherence translate into better clinical outcomes, such as a recent study that found improved viral suppression in patients with HIV enrolled in a DAT-based adherence intervention. ${ }^{21}$

DATs may be particularly relevant for rethinking TB care delivery, for a few reasons. First, improving TB medication adherence may have public health benefits, such as reduced rates of disease relapse, acquired drug resistance and transmission of infection. ${ }^{23}$ Second, unlike other diseases for which self-administered therapy is the standard of care, many TB programmes globally currently use DOT for monitoring. ${ }^{22}$ While some TB programmes may view DATs as a challenge to their existing DOT models, in many contexts, DATs may provide an alternative for 'observing' medication adherence, potentially making them more acceptable to patients with TB and HCPs than they are for other diseases, as is discussed further below. Finally, unlike chronic diseases that require lifelong treatment (eg, hypertension), TB treatment has a defined duration, such that monitoring the entire treatment course with DATs may be feasible.

DATs are being deployed for TB care in research and routine clinical practice in several countries, such as China, India and Belarus, prompting publication of a handbook by WHO regarding their use. ${ }^{23} \mathrm{~A}$ recent systematic review summarised the evidence on whether use of DATs improves TB medication adherence and treatment outcomes. ${ }^{24}$ This review found that SMS-based strategies have not been found to improve treatment completion rates in settings with suboptimal outcomes at baseline. The review found similarly high rates of treatment completion when comparing treatment monitoring by video DOT and in-person DOT in high-income country settings. In addition, the review found two studies suggesting that digital pillboxes may reduce missed doses and increase the probability of cure in some contexts. On the whole, however, that systematic review found the evidence supporting use of DATs for TB to be limited, and the authors suggest that more robust evidence is needed to understand how these technologies may impact patients and health systems. ${ }^{24}$

While that previous systematic review described the existing evidence on use of DATs for TB, this current narrative review has a different goal. We use the findings of that prior systematic review as a starting point for describing the landscape of existing technologies that are
Box 1 Search strategy and selection criteria

To better inform our narrative review, we searched PubMed for peerreviewed articles published between 1 January 2000 and 31 July 2017, with the terms referring to tuberculosis (eg, 'tuberculosis' OR 'TB' OR 'Mycobacterium tuberculosis') and terms that refer to digital adherence technologies generally (eg, 'adherence technology' OR 'mHealth' OR 'mobile technology' OR 'digital medication monitors' OR 'electronic monitors' OR 'remote observation') as well as specific technologies (eg, 'short messaging service' or 'cellphone' or 'smartphone' or 'digital pillboxes' OR 'electronic medication packaging' OR 'video DOT' OR 'ingestible sensors'). We only started our search after the year 2000 since these technologies are relatively new in the last 15 years. We selected case reports, qualitative studies, cohort studies, randomised trials and systematic reviews published in English. We also reviewed the references sections of these articles and sought advice from experts in the field to identify additional studies. We did not exclude any studies based on the methodology used or the study quality. We specifically excluded articles focused on technologies used to support medication adherence during treatement for latent TB infection.

currently being used and for highlighting critical gaps in research. Our review has been guided by a systematic search of the literature from 2000 to mid-2017 to ensure we cover the breadth of DATs currently being used in TB care (box 1). We first provide historical context for the use of DATs for TB and describe a conceptual framework that can inform their integration into clinical care. We then describe the variety of DATs that are being used for monitoring TB medication adherence and discuss key functions of DATs that have the potential to benefit patients and health systems. Finally, we highlight research priorities that could help to refine the testing and evaluation of DATs and create an evidence base to better understand their benefits and limitations for TB care.

\section{RETHINKING THE DOT MODEL}

The idea of direct observation of medication ingestion for TB emerged in the 1950s and 1960s from studies in Hong Kong and India. ${ }^{25}$ Some of the perceived strengths of direct observation included close monitoring of adherence, face-to-face interactions between patients and HCPs, and careful documentation of treatment records. In 1991, the World Health Assembly adopted the 'directly observed therapy, short-course' (DOTS) strategy. ${ }^{22}$ DOTS is a multipronged intervention for which direct observation of therapy is just one component. DOTS also included use of 'short-course' therapy (ie, 6 months), use of smear microscopy for diagnosis and systematic reporting of treatment outcomes. ${ }^{22}$ While treatment success rates globally improved under DOTS, ${ }^{22}$ the extent to which these improvements can be attributed to direct observation of therapy versus other aspects of the DOTS package are unclear.

Recent systematic reviews suggest that DOT does not achieve superior results compared with self-administered therapy (SAT) across a variety of outcomes-including 
treatment completion, ${ }^{10}{ }^{12}$ microbiological cure, ${ }^{10}{ }^{12}$ microbiological failure, ${ }^{11}$ disease relapse ${ }^{11}$ and acquired drug resistance. ${ }^{11}$ Interviews with patients with TB show that DOT may be associated with perceptions of low autonomy, inadequate confidentiality and stigma. ${ }^{9627} \mathrm{In}$ settings using facility-based DOT, frequent health facility visits may result in loss of income and employment. ${ }^{9} 2628$

DOT also raises challenges for health systems. Most DOT models assume that all patients require uniform monitoring-placing a high burden of supervision on HCPs-rather than stratifying patients by their level of non-adherence, so that HCPs can focus resources on the highest-risk patients. As a result of these challenges, in practice, DOT is poorly implemented, or not strictly adhered to in practice, in many TB programmes, especially where community DOT is used. ${ }^{2729-31}$ In light of the limitations of DOT, DATs have the potential to provide more patient-centric approaches for 'observing' pilltaking, ${ }^{32}$ to reduce financial burdens incurred by patients from frequent health facility visits and to identify non-adherent patients so HCPs can better focus their efforts. ${ }^{33}$

\section{LANDSCAPE AND FUNCTIONS OF TB ADHERENCE TECHNOLOGIES}

We describe select DATs that are being used in research or clinical care for TB in table 1 and figure 1 , along with details regarding SAT and DOT approaches for comparison. In SMS-based strategies, SMS texts remind patients to take medications; many approaches allow the patient to send a SMS response (ie, 'two-way') to indicate the dose has been taken. With 99DOTS, patients are issued TB medications in blister packs wrapped in a custom envelope. When a dose is dispensed, a hidden phone number is revealed on the inner envelope flap, prompting the patient to place a toll-free call to indicate a dose taken. ${ }^{34}$ In video DOT (VDOT), video conferencing via smartphone or computer allows HCPs to watch patients take medications, either synchronously (in real time) or asynchronously (at a different time using recorded video). Digital pillboxes have pre-programmed audiovisual reminders embedded in the pillbox. Opening and closing the box to access medications is recorded as a proxy for a dose taken. Ingestible sensors are microchips embedded in TB medications. Contact with a patient's gastric fluid after ingestion results in transmission of a signal to an adhesive monitor worn by the patient. From there, the information is transmitted to the patient's smartphone and then to a server, where HCPs can access dosing histories. Online supplementary appendix 1 provides more extensive details on each DAT. Below, we describe the key functions that DATs may perform in patient care (figure 2).

\section{Reminder function}

DATs provide reminders to patients, addressing forgetfulness, which is a common barrier to adherence. ${ }^{8}$ Forgetfulness is commonly thought of as a cognitive barrier to adherence, but it also reflects psychosocial and structural barriers faced by patients, such as forgetting doses due to alcohol use or working multiple jobs. Reminders may promote habit formation in pill-taking behaviour. ${ }^{1532}$ For most DATs, reminders take the form of SMS texts. Digital pillboxes have embedded audiovisual reminders (eg, glowing light and ringing sound), which have the benefit of prompting patients to the site where medications are stored.

\section{Digital observation of dose-taking}

Most DATs digitally 'observe' or record dose-taking, which is especially relevant in TB given the historical reliance on DOT. VDOT mimics DOT by allowing HCPs to view patients swallow pills. As with DOT, VDOT may raise concerns about patient autonomy-as patients may feel that being watched taking every dose is an invasion of privacy-although new technologies using automated facial recognition and pill identification could obviate the need for HCPs to watch every video. ${ }^{35}{ }^{36}$ For some DATs, such as two-way SMS-based strategies, 99DOTS and VDOT, an extra step is needed to report dose-taking that requires effort by the patient herself, such as responding to an SMS text, placing a phone call or getting on a video call. For these technologies, dosing histories are compiled based on patient responses, and inaccuracies in the dosing history may be introduced because patients could send SMS responses or phone calls without taking doses (ie, over-reporting) or take doses without sending SMS responses or phone calls (ie, under-reporting).

Digital pillboxes may minimise patient effort and the risk of HCPs accidentally mis-recording information on paper records because opening and closing the pillbox is digitally recorded as a 'dose taken'. However, there are other potential limitations to their accuracy-for example, if a patient removes medication blister packs from the pillbox, allowing doses to be taken without opening it. Ingestible sensors record pill-taking with relatively high sensitivity and specificity ${ }^{37} 38$; however, removal of the adhesive monitor that records information transmitted by the ingested sensors would result in under-reporting of adherence. For all of these strategies, inaccuracy in patient reporting may be reduced by patient education about the purpose and appropriate use of these devices.

\section{Compilation of dosing histories}

DATs compile patient dosing histories, which allow for 'real-time' or clinic visit-based adherence monitoring. In real-time approaches, doses are recorded right after the patient engages the technology (eg, opening the pillbox or sending an SMS response). HCPs remotely access these histories on a web-based interface, allowing them to identify non-adherence before the patient's next medication refill visit (figure 3). In clinic visitbased monitoring, HCPs access dosing histories during patient visits, for example by uploading the record to a computer from the patient's digital pillbox. HCPs can then counsel patients using dosing information 


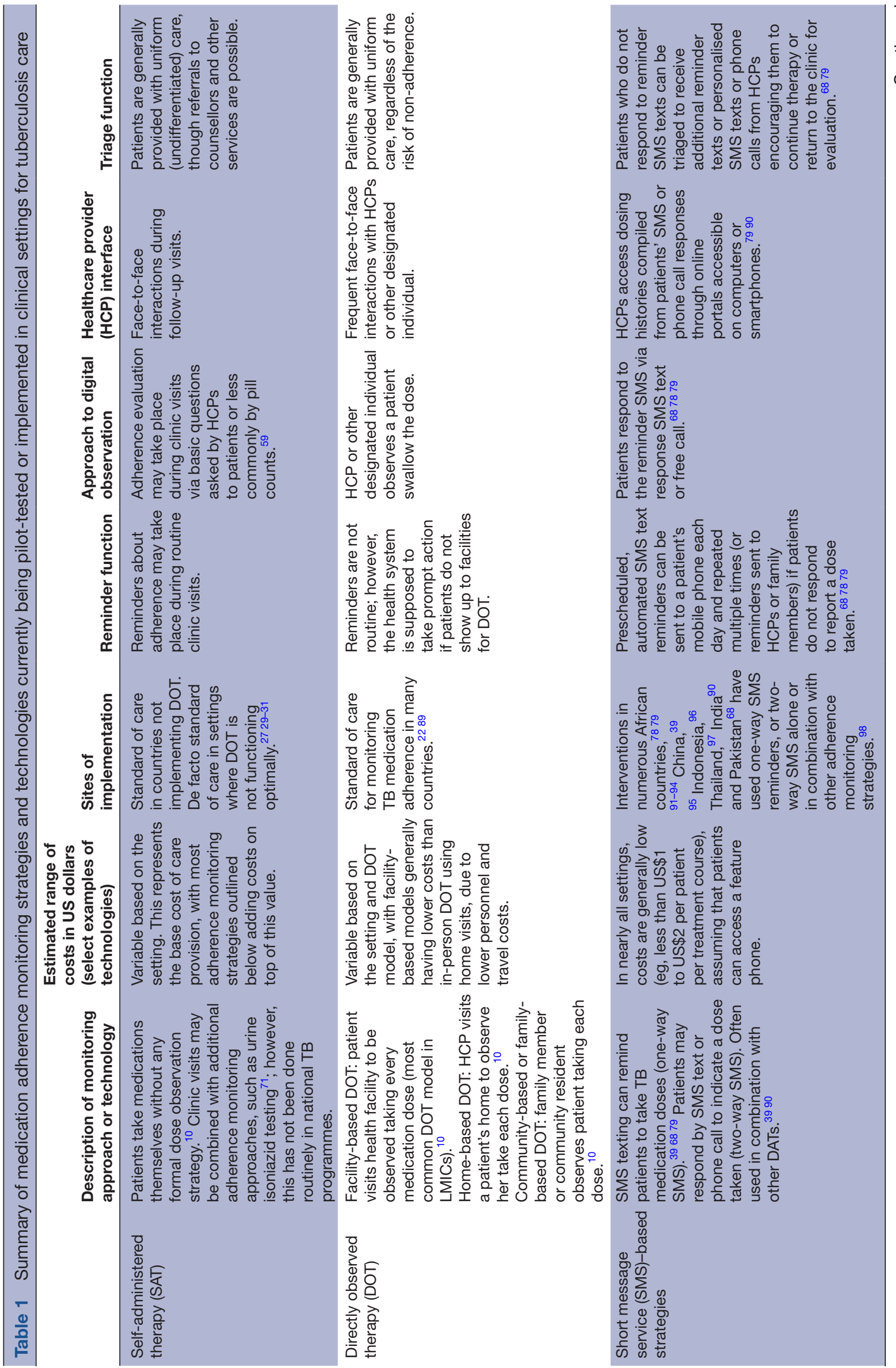




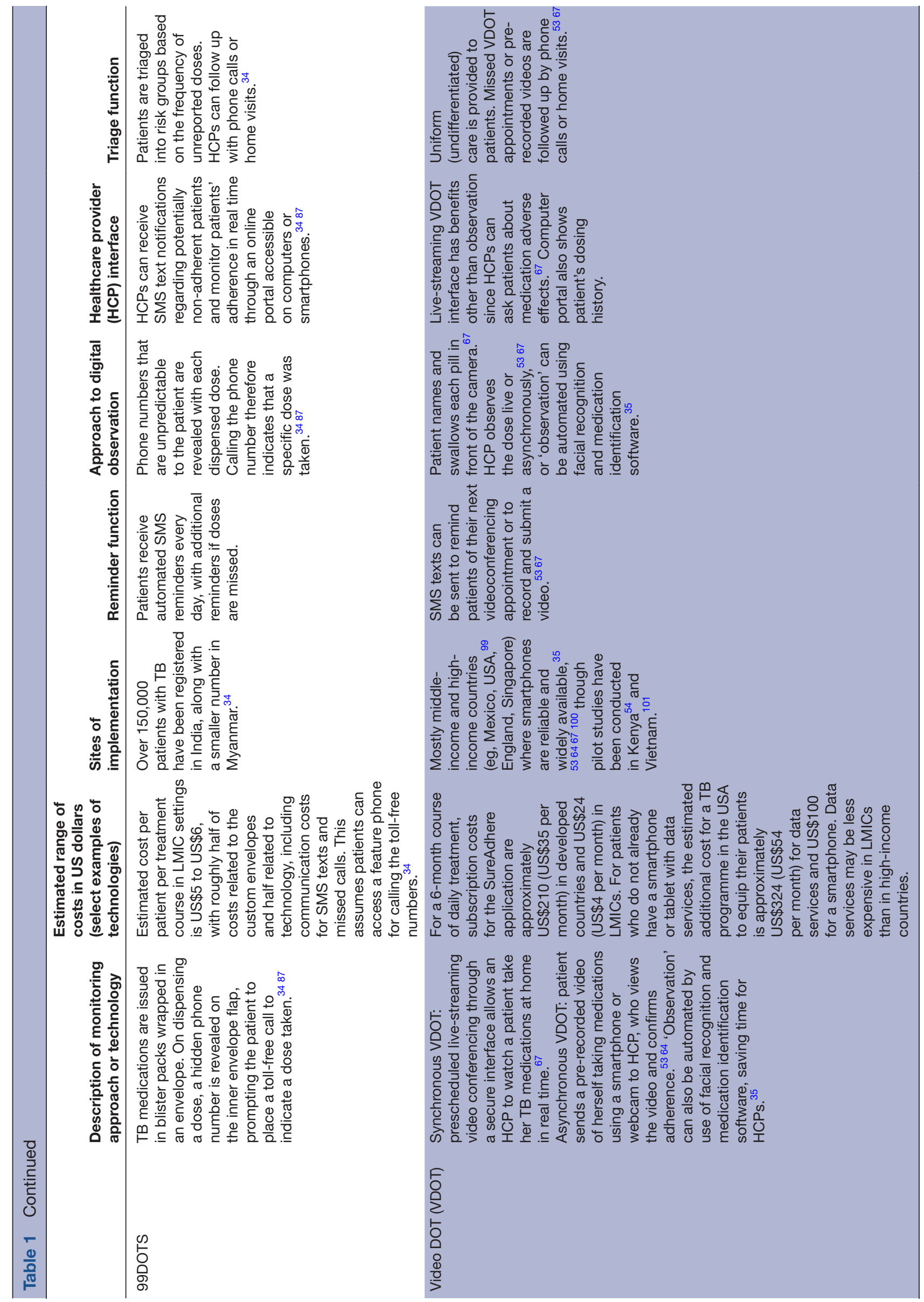




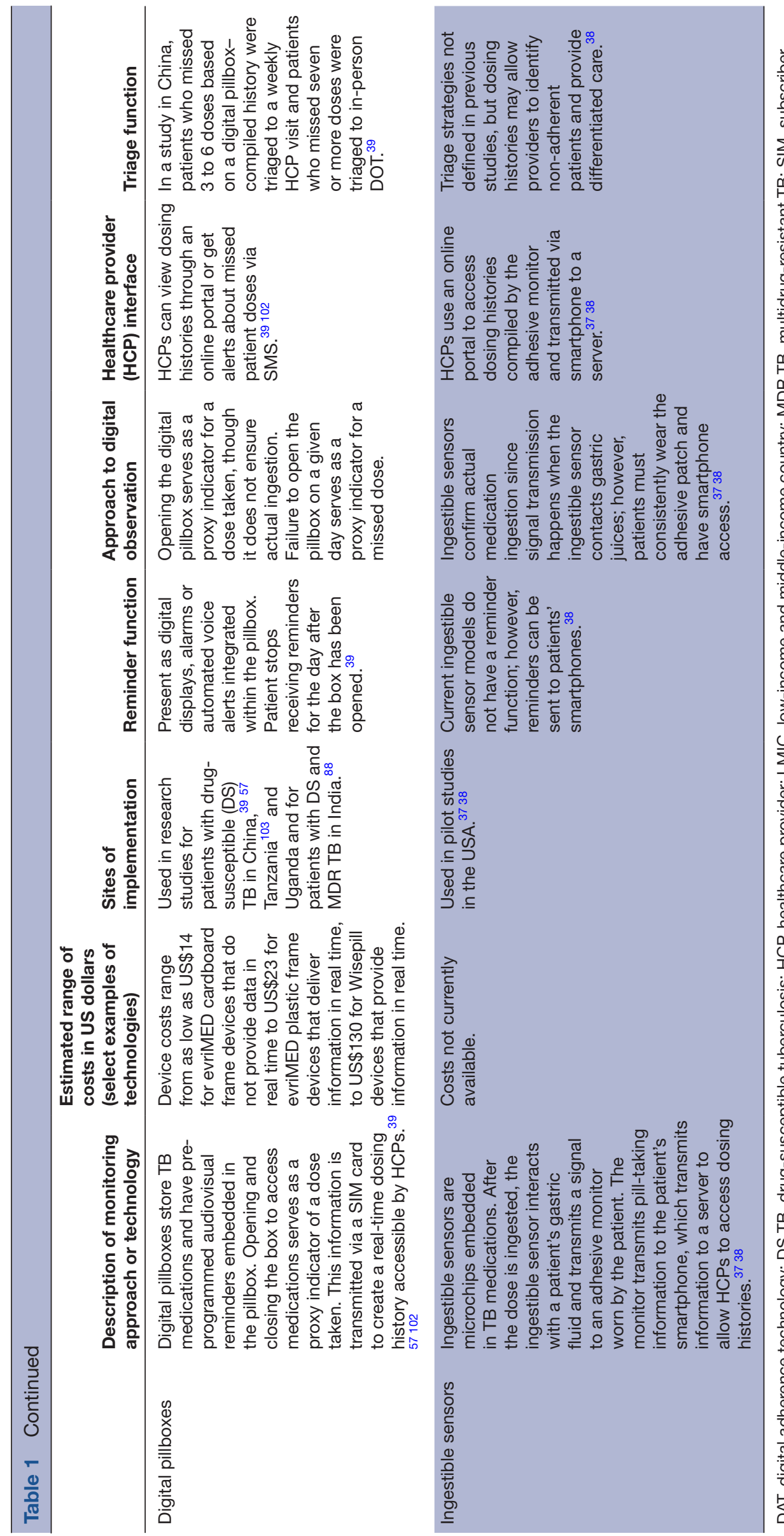




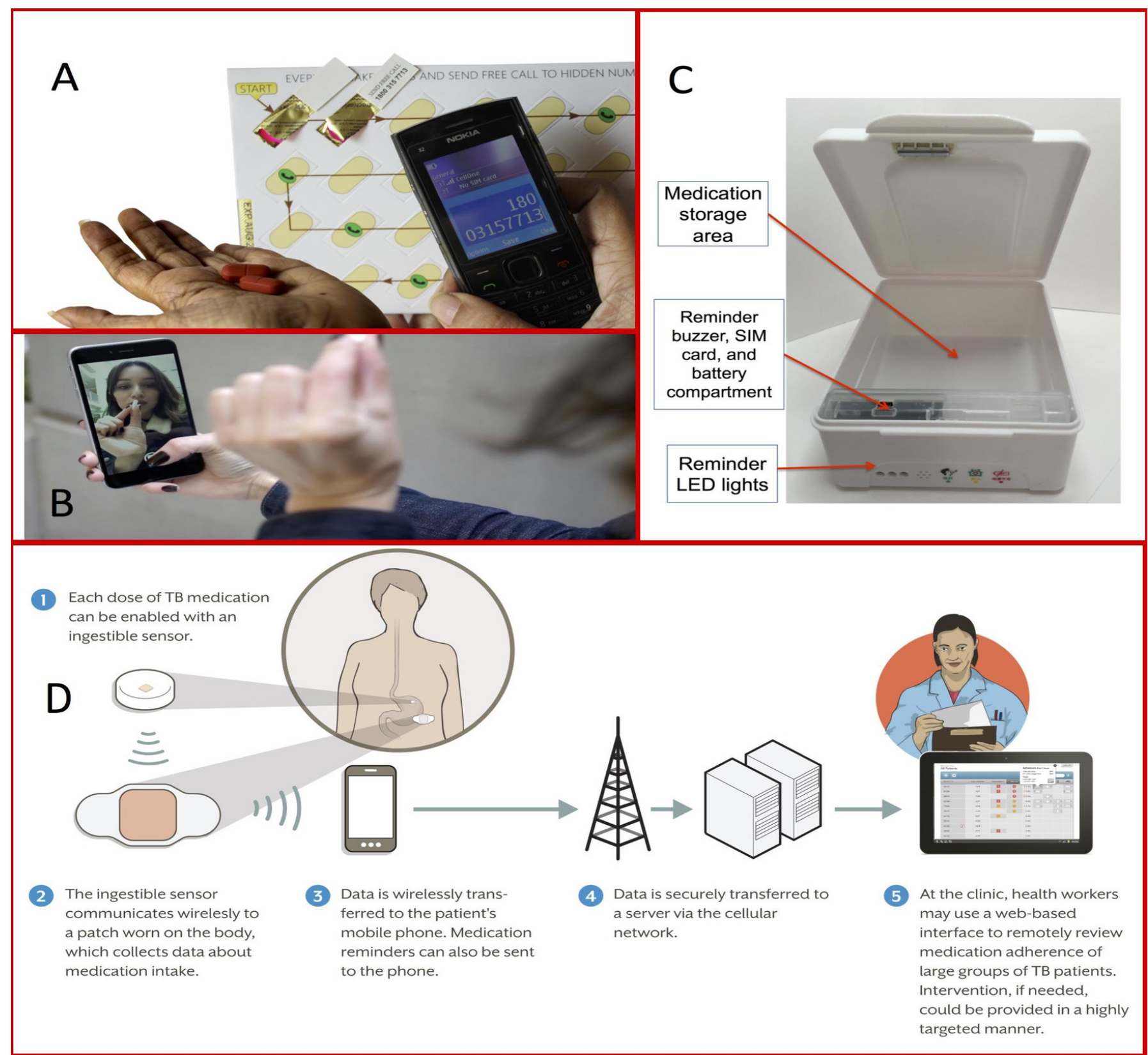

Figure 1 Examples of different adherence monitoring technologies. (A) 99DOTS, a feature phone-based adherence technology (with permission from Everwell Health Solutions); ${ }^{87}$ (B) SureAdherence, a video DOT strategy (with permission from SureAdherence Mobile Technologies); ${ }^{53}$ (C) evriMED, a digital pillbox (with permission from the Wisepill Technologies); ${ }^{88}$ (D) an ingestible sensor-based adherence monitoring approach (Source: Belknap et al. ${ }^{37}$ ). DOT, directly observed therapy; LED, lightemitting diode; SIM, subscriber identification module; TB, tuberculosis.

compiled since the last clinic visit. For example, in a study from China, at each clinic visit, the doctor reviewed the patient's dosing history for the prior month on a computer, discussed the reason for missed doses and switched patients who missed numerous doses to more intensive management strategies. ${ }^{39}$

\section{Triage and provision of differentiated care}

In contrast to DOT, in which patients are treated using a uniform approach, DATs can facilitate triage of patients based on different levels of adherence. Triage may be performed by HCPs during routine reviews of dosing histories. Alternatively, computer algorithms can be used to triage and alert HCPs about non-adherent patients to prompt early intervention, saving time for HCPs and potentially preventing patient loss to follow-up (figure 3). Triage can then facilitate escalation in the intensity of care for patients with a high level of non-adherence. For example, in a randomised trial of DATs in China, patients who missed three to six medication doses were switched to 'intensive management' consisting of weekly HCP visits to the patient's home. Those who missed $>7$ doses were switched to DOT. ${ }^{39}$ 


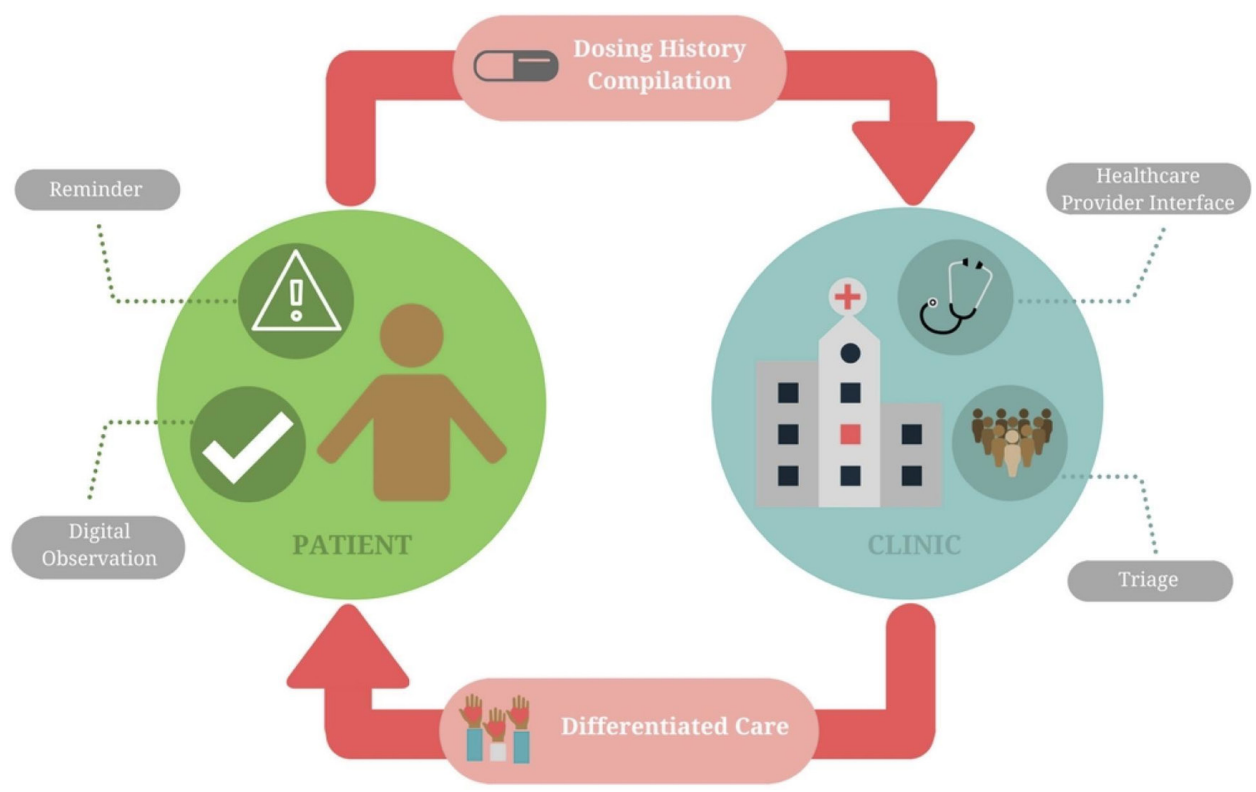

Figure 2 Functions that digital adherence technologies (DATs) can play to reinforce patient medication adherence and facilitate monitoring and triage of patients by health systems. 'Differentiated care' refers to providing different intensities and types of care based on a patient's level of medication adherence as measured by the DAT.

While DATs may help identify poor adherence, a careful clinical evaluation is needed to address its causes, which are often complex and may include psychosocial, treatment-related and health system-related barriers. As such, identification of non-adherence using DATs should ideally be viewed as the starting point for more intensive face-to-face engagement with patients to understand their specific reasons for non-adherence, so that individualised packages of care can be provided (ie, 'differentiated care'). By triaging patients, HCPs may be able to spend more time on these high-risk patients to address their needs. Triage and differentiated care have the potential to focus limited resources on higher-risk patients, which may improve the efficiency of care delivery.

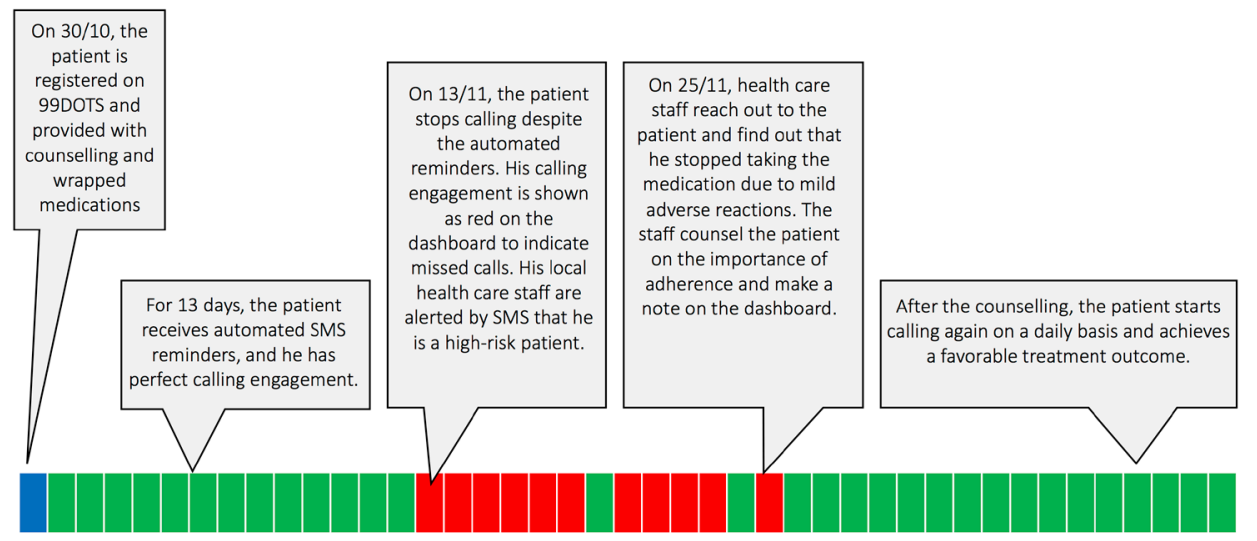

Figure 3 Example of how digital adherence technologies involving daily reporting of dose-taking could potentially facilitate earlier identification and intervention to address medication non-adherence. The 99DOTS model is used for illustrative purposes. Each box represents a calendar day on the dashboard viewed by healthcare providers. Green boxes represent doses that were 'called in' on a given day and red boxes represent doses that were not called in. SMS, short messaging service.

\section{RESEARCH PRIORITIES AND EXISTING EVIDENCE Acceptability and ethical questions}

Acceptability may differ based on the type of DAT and the context in which it is deployed. Development of DATs should ideally follow a participatory approach that iteratively incorporates views of users and stakeholdersincluding patients with $\mathrm{TB}$, caregivers and HCPs-in order to improve acceptability. ${ }^{40}$ Models that have been shown to predict acceptance of health technologies, such as the Unified Theory of Acceptance and Use of Technology and the Technology Acceptance Model, can guide evaluations of DAT acceptance. ${ }^{42-44}$ In general, these models evaluate 'ease of use' (ie, how easily users are able to learn the DAT), 'perceived usefulness' (ie, 
whether users think it is valuable for TB care) and the availability of infrastructure to support their use. Frameworks have also been proposed for evaluating ethical aspects of DATs. ${ }^{4546}$ These frameworks emphasise evaluation of patient autonomy (including concerns about 'surveillance'), ${ }^{47}$ privacy and confidentiality, stigma and intrusiveness, and trust between patients and HCPs. ${ }^{45} 46$

Patient literacy-with regard to language (for two-way SMS), using a feature phone (for two-way SMS or 99DOTS) or using a smartphone (for VDOT or ingestible sensors)—should be assessed in any evaluation of acceptability. In populations with low language literacy, providing reminders in local languages or voice messages (rather than SMS texts) may expand the reach of some DATs. Researchers should also assess optimal programming of a DAT's reminder function. Reminders that are programmed for the wrong times-for example when patients are sleeping or at work-may make it difficult to respond in a timely manner. Overly frequent reminders may result in patients opening pillboxes just to shut off reminders or discarding SMS texts before they are read. ${ }^{39}$

Maintaining privacy of a patient's TB diagnosis is an important aspect of acceptability ${ }^{45}$ because stigma can result in discrimination or negatively affect a patient's coping capacity. ${ }^{64}$ Unintentional disclosure of disease status could occur if other individuals see a patient's SMS texts, ${ }^{49}$ medication envelopes (for 99DOTS), digital pillbox, ${ }^{46}$ video observation session or adhesive monitor (for ingestible sensors). This may especially be a problem in settings where there are high levels of shared cellphone use within families. Confidentiality may be breached if unauthorised persons access digital adherence data, which may particularly be a problem in settings where regulations for electronic health data are lacking or not enforced. Understanding how cultural characteristics shape patient tolerance for loss of privacy and confidentiality may enable user-centred design. For example, use of a password allowing SMS texts to be read only by the patient may be an option to protect privacy in some settings. ${ }^{50}$

Studies of SMS texting reminders (conducted in Peru, Argentina, Uganda, Pakistan and China), ${ }^{51-52}$ VDOT (conducted in the USA, Mexico and Kenya), ${ }^{53-56}$ and a digital pillbox (conducted in China) ${ }^{57}$ have generally shown high acceptability of these technologies by patients in surveys and qualitative interviews. Notably, however, one study from the USA suggested low acceptability $(33 \%)$ of SMS dose reminders, and SMS response rates in actual implementation are often lower (eg, 60\%-80\% at best) than indicated in acceptability surveys. ${ }^{52} 58$

\section{Feasibility}

Despite widespread cellular ownership in many low-income and middle-income countries (LMICs), there is still considerable variability in levels of cellphone access and cellular network coverage by country as well as potentially within specific subpopulations within countries. As such, feasibility challenges remain in some settings, including the following for cellphone-based strategies: limited cellphone ownership, use of shared cellphones, low cellphone literacy, poor audio or video quality, ${ }^{59}$ poor cellular network connexions, technical failures preventing receipt of SMS texts, electricity outages and changing phone numbers. ${ }^{496}$ Feasibility challenges for digital pillboxes may include battery failure, device malfunction (leading to data losses) and problems related to cellular networks. In general, there have been ongoing improvements in the feasibility of DATs. For example, the battery life of digital pillboxes has improved to as long as 6 months. Some devices temporarily store data in flash memory or use GPRS (General Packet Radio Service) to maintain data in transit until acknowledgement of receipt by the web server, reducing data losses due to power failures or poor cellular connectivity. ${ }^{61}$

Surveys conducted in Argentina and China suggested feasibility of SMS-based strategies due to high cellphone ownership and literacy. ${ }^{62}{ }^{52}$ However, a recent study from Peru highlights that, although cellphone access may be high in the general population, access may be considerably lower in patients who suffer from TB and in particular for patients with TB with poor treatment outcomes. ${ }^{63}$ Implementation studies from high-income and middle-income countries (USA, Canada, Belarus, Mexico) have suggested that VDOT has high feasibility, ${ }^{53}$ 64-66 though some patients had to be shifted back to in-person DOT $^{53}$ and low video or audio quality sometimes made dose observation difficult. ${ }^{65}{ }^{67}$ Ingestible sensors had high feasibility in studies conducted in the USA and Mexico, with $>95 \%$ of sensor signals detected after ingestion. ${ }^{37} 38$

In some settings, a more fundamental barrier to implementation of DATs may be health system resource constraints, such as lack of computers in clinics to view dosing histories and shortages of HCPs who could act on this information to address non-adherence in high-risk patients.

\section{Accuracy of digital observation}

Each DAT has limitations outside of technical failures that may reduce its accuracy for verifying true medication adherence, resulting in over-reporting (ie, false-positive signals in the dosing history) or under-reporting (ie, false-negative signals). Strategies relying on self-reporting via SMS texts or phone calls are at particular risk for under-reporting of adherence if patient engagement wanes due to 'technology fatigue', as illustrated in a two-way SMS intervention in Pakistan (figure 4). ${ }^{68}$ Digital pillboxes may under-report adherence if medication blister packs are taken out of the pillbox so that doses can be taken without opening the device or from device non-use due to patient travel or stigma. Ingestible sensors could under-report true adherence if the adhesive monitor used to record signals from the ingestible sensors is removed. Alternatively, over-reporting may occur if patients indicate adherence via SMS texts, phone 


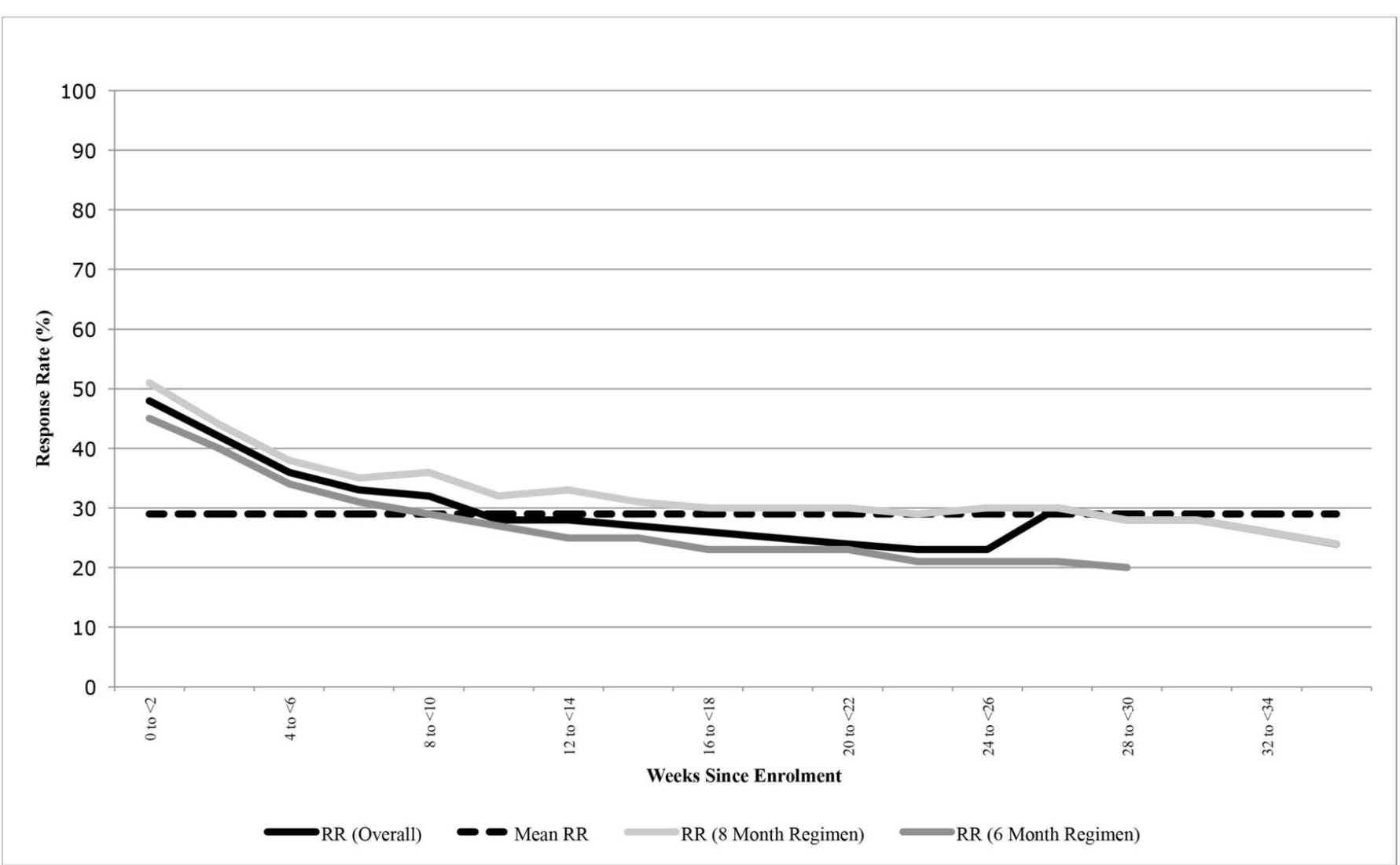

Figure 4 Example of 'technology fatigue'. Patient response rates to short messaging service (SMS) texts to indicate dose ingestion declined throughout the course of tuberculosis therapy in a study of a two-way SMS intervention in Pakistan, reducing the accuracy of this monitoring approach. Source: Mohammed et al. ${ }^{68} \mathrm{RR}$, response rate.

calls or pillbox openings without taking the doses, though such behaviour often wanes over time. ${ }^{69}$

As such, evaluation of accuracy in research studies is critical to ensure that a DAT provides a reasonable proxy of true adherence. When conducting such research evaluations, careful use of nomenclature is important because it is easy to conflate the quality of a patient's medication adherence with the quality of her engagement with the DAT, even though these two are not the same. Building on nomenclature recommendations for describing medication adherence, ${ }^{70}$ we suggest parallel nomenclature for describing a patient's engagement with DATs (table 2).

Evaluations of accuracy generally compare DAT-compiled dosing histories to an alternative adherence metric to determine the DAT's sensitivity, specificity, positive predictive value and negative predictive value for true adherence. These alternative metrics include biological tests of drug ingestion (eg, urine testing for isoniazid content), pill counts, refill data or concurrent monitoring

Table 2 Suggested nomenclature for describing medication adherence and engagement with digital adherence technologies

\section{Taxonomy for describing adherence to medications Taxonomy for describing engagement with digital adherence (from Vrijens et $\mathrm{al}^{70}$ ) technologies}

Initiation: time point when the first dose of medication is taken

Dosing implementation: correspondence between patient's actual dosing and the prescribed dosing regimen

Persistence: length of time between initiation and last dose

Discontinuation: time point when the patient takes her last dose
Starting: time point when engagement with technology begins (eg, first SMS text response, phone call, digital pillbox opening etc)

Technology participation: correspondence between expected daily engagement with the technology and actual daily engagement

Duration of engagement: length of time between starting and stopping of engagement with the technology

Stopping: time point when the patient has the last recorded engagement with the technology (eg, last SMS text response, phone call, digital pillbox opening etc)

SMS, short messaging service. 
with another DAT, such as use of a 'silent' digital pillbox (ie, reminder function disabled) in patients using feature phone-based strategies (online supplementary appendix 1 for details). There is no 'gold standard' metric since all comparators, including biological tests, have their own inaccuracies; examples include interindividual and intraindividual variation in drug absorption and metabolism. ${ }^{71-73}$

We recommend using multiple comparators to gain the fullest understanding of a DAT's accuracy. For example, urine isoniazid testing provides a 'snapshot' of dose-taking that can be compared with DAT dosing histories over the prior 24-72 hours, while medication refills provide data on persistence with therapy that can be compared with longer-term dosing histories. Collecting biological test or pill count data during unannounced home visits (ie, without prior notice) may help to minimise the 'Hawthorne effect' - that is, short-term changes in patient adherence or DAT engagement in anticipation of clinic visits. ${ }^{74}$ In patients concurrently taking medications for other comorbid conditions, such as HIV or diabetes, it is also important to assess the impact of a DAT on medication adherence for all conditions, if possible.

Few studies have rigorously evaluated the accuracy of DATs. One study in China found high correlation between dosing histories from a digital pillbox and urine rifampin test results. ${ }^{75}$ A small pilot study in South Africa found high correlation between dosing histories from digital pillboxes and pill counts conducted for patients taking therapy for MDR TB and HIV. ${ }^{76}$ Studies have suggested that VDOT and ingestible sensors may be more accurate than in-person DOT since they are able to 'observe' a greater proportion of doses, especially on weekends and holidays. ${ }^{3867}$

\section{Adherence and treatment outcomes}

Health outcomes-including medication adherence, treatment success and post-treatment recurrence free-survival-are the most important indicators of DATs' impact on TB care (table 3, online supplementary appendix 1). When assessing health outcomes, especially in randomised trials or quasi-experimental studies, it is important to identify the appropriate comparator representing the baseline standard of care against which a DAT-based care model will be compared. In many settings, DAT-based models should be compared against DOT, recognising that DOT models may vary from setting to setting, including facility-based DOT, in-person DOT, and community or family DOT (table 1 ). In other settings, SAT may be the standard of care, though SAT models may also vary based on the frequency of patient-provider contact or medication refills (eg, biweekly, monthly etc). When DAT-based care models are compared against SAT models, we recommend that the SAT model should at minimum include a protocol for patient outreach and engagement if a patient misses a medication refill date since missing a refill may be suggestive of non-adherence. Comparing DAT-based models to SAT models that follow
Table 3 Example of information that can be collected to evaluate the impact of medication adherence technologies on treatment outcomes

\begin{tabular}{|c|c|}
\hline Outcome & Potential definitions \\
\hline \multirow[t]{3}{*}{$\begin{array}{l}\text { Medication } \\
\text { adherence (ie, dosing } \\
\text { implementation } \\
\text { and persistence on } \\
\text { therapy) }\end{array}$} & $\begin{array}{l}\text { Proportion of all expected doses } \\
\text { that were missed during the full } \\
\text { treatment course }{ }^{\star} \text { (continuous } \\
\text { outcome) }\end{array}$ \\
\hline & $\begin{array}{l}\text { Proportion of patient months with } \\
>\text { X\%† of expected doses missed } \\
\text { (continuous outcome) }\end{array}$ \\
\hline & $\begin{array}{l}\text { Proportion of patients who } \\
\text { completed therapy with }<\mathrm{X} \% \\
\text { of expected doses missed over } \\
\text { the full treatment course (binary } \\
\text { outcome) } \dagger\end{array}$ \\
\hline Treatment interruptions & $\begin{array}{l}\text { Proportion of patients who } \\
\text { completely interrupt tuberculosis } \\
\text { (TB) therapy for a short time period } \\
\text { (eg, >1 month) or who are formally } \\
\text { lost to follow-up (eg, }>2 \text { months) } \\
\text { (binary outcome) }\end{array}$ \\
\hline \multirow[t]{3}{*}{ Treatment success } & $\begin{array}{l}\text { Proportion of patients who } \\
\text { achieved cure or treatment } \\
\text { completion (binary outcome) }\end{array}$ \\
\hline & $\begin{array}{l}\text { Proportion of patients who } \\
\text { achieved cure or treatment } \\
\text { completion without extension of } \\
\text { treatment duration due to non- } \\
\text { adherence (binary outcome) }\end{array}$ \\
\hline & $\begin{array}{l}\text { Mean or median number of } \\
\text { medication refills per patient as } \\
\text { a proxy of months of treatment } \\
\text { completed (continuous outcome) }\end{array}$ \\
\hline $\begin{array}{l}\text { Post-treatment } \\
\text { tuberculosis } \\
\text { recurrence-free } \\
\text { survival }\end{array}$ & $\begin{array}{l}\text { Proportion of patients who } \\
\text { complete TB therapy and achieve } \\
\text { 1-year recurrence-free survival } \\
\text { (binary outcome) } £ \S\end{array}$ \\
\hline
\end{tabular}

*An 'ideal' length of therapy could be used for assessing the number of expected and missed doses-for example, 182 expected treatment days for patients on daily therapy for drugsusceptible TB (see online supplementary appendix 1). †Threshold of the percentage of expected doses missed can vary depending on baseline rates of adherence (eg, greater than or less than $10 \%, 20 \%$, etc).

$\ddagger$ That is, patients who achieve treatment completion and do not experience post-treatment TB recurrence or death.

$\S$ Follow-up times can vary, though we recommend a minimum of 6 months of post-treatment follow-up.

up on missed refills may reveal whether providing HCPs with more granular day-to-day real-time information from DATs (a proposed 'value-add' of some of these technologies) actually leads to better outcomes than a more crude but simple approach of following up promptly on patients who miss their medication refill dates.

While a variety health outcomes may be assessed, improvements in surrogate endpoints that DATs may 
affect directly (eg, medication adherence) may not always translate into impacts on longer-term outcomes (eg, treatment success or recurrence-free survival) because improvements in longer-term outcomes often require addressing multiple aspects of quality of care, such as ensuring early diagnosis, drug-susceptibility testing, appropriate medication dosing and so on. ${ }^{77}$

As such, we recommend that researchers initially focus on assessing whether DATs improve TB medication adherence. In settings where DATs transform care considerably (eg, shifting away from facility-based DOT), it is also important to routinely monitor treatment success rates to ensure that these outcomes remain comparable, at minimum. When evaluating treatment outcomes, it is important that the entire "package of care' involving the DAT be well defined-including approaches for triaging patients based on dosing histories and the interventions that will be delivered to non-adherent patients.

The study design used to evaluate health outcomes depends on the study goal and resources available. Cohort studies can evaluate whether health outcomes are achieving minimal standards recommended by $\mathrm{TB}$ programmes; however, this design is not optimal for understanding whether DATs themselves have contributed to improvements or deterioration in outcomes compared with prior standards of care. Historical programmatic data may be vulnerable to inaccuracy and provide a poor baseline for understanding the relative benefits of DATs.

Quasi-experimental designs that evaluate outcomes prior to and after rollout of a DAT-based intervention may provide more helpful evidence regarding changes in outcomes; however, these findings may be confounded by other concurrent changes to TB care. Trials using randomisation of patients or larger units of care (eg, health facilities) to DAT-based interventions provide the most rigorous evidence of impact on health outcomes.

As highlighted in a recent systematic review, ${ }^{24}$ evidence regarding the impact of DATs on TB outcomes remains limited. Randomised trials of SMS strategies have not shown improvements in adherence or treatment success, ${ }^{39526878}$ with the exception of a Kenyan study that included prompt engagement by HCPs for patients who did not report pill-taking via SMS. ${ }^{79}$ One South African study of digital pillboxes found improved TB cure rates compared with historical controls, though cure rates in the controls were poor compared with international standards. ${ }^{80}$ A more rigorous cluster-randomised trial in China found that patients in study arms monitored with a digital pillbox had reduction in medication non-adherence (ie, patient-months with $>20 \%$ of doses missed) compared with the standard of care ${ }^{39}$ Cohort studies of patients monitored by VDOT in the USA and Australia have shown comparable outcomes to routine in-person DOT. $^{5967}$

\section{Costs and cost-effectiveness}

In settings with high baseline rates of treatment success, the primary benefit of DATs may be in reduction of costs and patient and HCP burdens associated with existing DOT or SAT models. ${ }^{9} 2628$ The 'costs' of implementation should be defined broadly from a societal perspective to include material components, communication costs, new personnel, changes in time use by existing HCPs, and changes in costs and other burdens borne by patients (table 4). Micro-costing techniques (eg, time and motion studies) may allow estimation of costs resulting from changes in time and work use by HCPs. ${ }^{81}$

Estimating an incremental cost-effectiveness ratio requires data on the difference in costs between the existing care model and a DAT-based model, as well as the difference in health outcomes between the two models, ideally represented as disability-adjusted life years. As such, costing studies should collect data on the costs of existing care models (eg, DOT or SAT), and concurrent studies estimating health outcomes for the different models would be required for a formal cost-effectiveness analysis. Modelling may be required to estimate the cost-effectiveness of DATs for TB programmes a national level. ${ }^{21} 82$ Cost-effectiveness of different DATs may vary between high-income countries (where personnel costs are high) and LMICs (where personnel costs are relatively low).

A US study of VDOT factoring in HCP and patient time and travel costs estimated an average cost savings of US $\$ 2248$ per patient per TB treatment course as compared with home-based DOT $^{83}$; however, an Australian study found that VDOT would be more cost-effective than in-person DOT only with scale-up or decreased technology costs. ${ }^{59}$ A South African study of digital pillboxes estimated a return on investment of $23 \%$ over 5 years, largely from cost savings due to improved treatment outcomes; however, this study assumed very poor treatment success rates seen with historical controls. ${ }^{80}$

\section{Triage strategies and provision of differentiated care}

Most DATs aim to identify non-adherent patients who may require additional support to improve adherence or prevent loss to follow-up. Poorly designed interventions for addressing non-adherence may therefore attenuate the beneficial impacts of DATs. HCPs might find themselves in a 'data glut', without the guidance or capacity to act productively on the rich real-time data compiled by DATs. A critical question hovering over DAT-based strategies is: how can the data-rich dosing histories compiled by DATs be best leveraged to provide effective individualised (or 'differentiated') care that will improve adherence?

Addressing this question will require researchers and HCPs to think beyond the technologies to understand the medical, psychosocial, cultural, structural and health system barriers that contribute to non-adherence. ${ }^{7}$ Such research could inform the development (by iterative testing) of intervention packages targeted towards patients with different levels of risk for non-adherence. 


\begin{tabular}{|c|c|c|c|}
\hline Material costs & Communication costs & Personnel costs & Patient-related costs \\
\hline $\begin{array}{l}\text { Devices if provided } \\
\text { to the patient (digital } \\
\text { pillboxes, feature } \\
\text { phones or smartphones) } \\
\text { Platforms for } \\
\text { visualising dosing } \\
\text { histories by healthcare } \\
\text { providers (computers, } \\
\text { smartphones) } \\
\text { Data servers } \\
\text { Medication envelopes } \\
\text { (for 99DOTS) } \\
\text { Ingestible sensors }\end{array}$ & $\begin{array}{l}\text { SMS text costs } \\
\text { Direct phone calls to } \\
\text { patients (including } \\
\text { call centres for some } \\
\text { strategies) } \\
\text { Video communication/ } \\
\text { internet costs }\end{array}$ & $\begin{array}{l}\text { Costs of new personnel for } \\
\text { managing information technology } \\
\text { or other tasks such as packing } \\
\text { medications blister packs in } \\
\text { envelopes (for 99DOTS) } \\
\text { Cost of new counsellors or } \\
\text { other providers in some settings } \\
\text { to facilitate more intensive } \\
\text { management of barriers to } \\
\text { adherence (eg, treatment literacy, } \\
\text { depression counselling, treatment } \\
\text { of alcohol use disorder) in } \\
\text { differentiated care models } \\
\text { Changes in resource or time use } \\
\text { by existing healthcare personnel } \\
\text { due to decreased time spent in } \\
\text { direct observation, reduced travel } \\
\text { costs with elimination of home } \\
\text { DOT, or increased time spent on } \\
\text { troubleshooting DATs or managing } \\
\text { data entry }\end{array}$ & $\begin{array}{l}\text { Potentially reduced costs } \\
\text { of travel and decreased } \\
\text { time spent on visiting } \\
\text { healthcare facilities for } \\
\text { direct observation (under } \\
\text { facility-based DOT) } \\
\text { Potential time saved } \\
\text { by not having wait for a } \\
\text { healthcare provider to visit } \\
\text { (under home DOT) }\end{array}$ \\
\hline
\end{tabular}

DAT, digital adherence technology; DOT, directly observed therapy; SMS, short messaging service.

Examples of adherence intervention packages that leverage DAT dosing histories exist for other diseases, such as HIV. ${ }^{21}$ These packages of care could screen for and address common causes of non-adherence in each setting, which may include poor treatment literacy, ${ }^{84} \mathrm{~TB}$ medication toxicities, ${ }^{5}$ depression, ${ }^{58586}$ substance use disorders, ${ }^{4}$ financial burdens ${ }^{7}$ or difficulties travelling to health facilities. ${ }^{7}$ By triaging patients, DATs may allow HCPs to focus more time and attention on a smaller group of high-risk patients, and ancillary personnel, such as dedicated counsellors or psychologists, could potentially help address their more intensive needs. To date, research evaluating triage and differentiated care strategies has been limited.

\section{CONCLUSION}

DATs have the potential to transform TB care delivery by facilitating more patient-centric strategies for monitoring adherence, while providing HCPs with real-time data that can enable patient triage. The current DAT landscape includes a diverse array of technologies that are in development, undergoing pilot testing or being rolled out at scale as part of clinical care. These DATs employ different devices, reminder functions and approaches for compiling dosing histories, which may contribute to differences in their acceptability, implementation costs and the financial resources required for rollout in different settings.

As such, there is no 'perfect' DAT that will work optimally in every setting (especially high-income countries as compared with LMICs) or even for every patient in a single setting. Development of software platforms that can compile dosing histories from multiple DATs may allow HCPs to monitor TB patients who have varied needs using different technologies in the same setting. For example, in India, a single platform has been developed that compiles dosing histories from multiple DATs, which allows patients with cellphones to be monitored using 99DOTS while those without cellphones can be monitored using digital pillboxes. In addition, combining information from DAT-based strategies with data from non-DAT monitoring approaches in clinical practicesuch as urine isoniazid testing or medication refill datamay be helpful in cases where patient engagement with the technology is suboptimal, resulting in inaccurate dosing histories.

Research is needed to understand the impact of these technologies on patients and health systems and to inform approaches for provision of differentiated care. Outside of pilot data on $\mathrm{VDOT}^{67}$ and ingestible sensors ${ }^{37} 38$ in high-income settings and on digital pillboxes in China and South Africa, ${ }^{756}$ little is known about the accuracy of DATs for measuring adherence in patients with TB, especially with larger-scale implementation in LMICs and for patients concurrently taking medications for comorbid conditions such as HIV or diabetes. Most importantly, more robust data are required on DATs apart from two-way SMS to determine whether they have positive impacts on health outcomes, especially in highTB-burden LMICs. 
Finally, while studies in TB have heavily focused on the DATs themselves, less emphasis has been placed on understanding how DATs can be leveraged to provide differentiated care to patients who require more intensive support to achieve optimal treatment outcomes. Little work has been conducted to understand the causes of medication non-adherence in different populations of patients with $\mathrm{TB}$, so that clinical protocols can be designed to help HCPs screen for and address these causes. If the rollout of DATs also stimulates rethinking of the HCP-patient interaction, then DATs have the potential to move 'care' into non-traditional spaces (such as the home or the workplace) and to serve as an extension of the health system. ${ }^{32}$ Otherwise, DAT-based monitoring strategies run the risk of overly focusing on 'observation' and replicating some of the paternalistic aspects of existing DOT approaches. In our opinion, if DATs are viewed as tools for enhancing (rather than limiting) faceto-face human interactions, then they will have stronger potential for transforming TB care delivery by creating truly patient-centric models of care.

\section{Author affiliations}

${ }^{1}$ Department of Public Health and Community Medicine and Tufts Center for Global Public Health, Tufts University School of Medicine, Boston, Massachusetts, USA ${ }^{2}$ Division of Geographic Medicine and Infectious Diseases, Tufts Medical Center, Boston, Massachusetts, USA

${ }^{3}$ Division of Infectious Diseases, Brigham and Women's Hospital, Boston, Massachusetts, USA

${ }^{4}$ Department of Information Technology, Mbarara University of Science and Technology, Mbarara, Uganda

${ }^{5}$ McGill International TB Centre, McGill University, Montreal, Quebec, Canada

${ }^{6}$ The Fenway Institute, Fenway Health, Boston, Massachusetts, USA

${ }^{7}$ Division of Infectious Diseases, Beth Israel Deaconess Medical Center, Boston, Massachusetts, USA

${ }^{8}$ Department of Social and Behavioural Research, National Institute for Research in Tuberculosis, Chennai, Tamil Nadu, India

${ }^{9}$ Center for Global Health, Massachusetts General Hospital, Boston, Massachusetts, USA

Acknowledgements We appreciate critical feedback on the manuscript from Bruce Thomas (The Arcady Group) as well as from the three anonymous reviewers for the journal. William Thies, Andrew Cross and Nakull Gupta (Everwell Health Solutions) provided cost data for 99D0TS and SMS strategies and a photo of 99D0TS and the 99D0TS dashboard for healthcare providers. Richard Garfein (University of California at San Diego) provided cost data and a photo of the SureAdherence Video DOT technology. Cost data for evriMED and Wisepill technologies and a photograph of the evriMED digital pillbox were obtained from Wisepill Technologies and Bruce Thomas (The Arcady Group). The corresponding author had access to all the data in the study and had final responsibility to submit for publication.

Contributors RS, JH, AM and LdM conceived the report. RS, LdM, AM and JH wrote the first draft. LdM and RS conducted the literature search and designed the figures. KHM, MP, JH, BET and AM revised, edited and provided critical additions to the manuscript. All authors approved the final version.

Funding RS is supported by a grant from the Bill and Melinda Gates Foundation via The Arcady Group (OPP1154665) and a Doris Duke Clinical Scientist Development Award. He acknowledges prior support for this work through a development award from the Harvard Center for AIDS Research (5P30AI06035413) and a Harvard Catalyst KL2/CMERIT Award (KL2 TR001100). LdM was supported by a grant from the Harvard Center for AIDS Research (5P30Al06035413). AM and JH are supported by an Emerging Global Leader Award from the Fogarty International Center (K43TW010388) and a mid-career development award from the National Institute of Mental Health (K24MH114732), respectively. BET conducted research on adherence technologies supported by the Bill \& Melinda
Gates Foundation (OPP1154670). MP holds a Canada Research Chair award from the Canadian Institutes of Health Research.

Disclaimer The funding sources had no role in the conception, design, analysis or writing of the report, or in the decision to submit it for publication.

Competing interests BET and RS are currently conducting research evaluating the implementation of 99D0TS and evriMED (a digital pillbox) in India, supported by the Bill and Melinda Gates Foundation; neither has any financial interest in these technologies. AM and $\mathrm{JH}$ are currently conducting research on the Wisepill device (a digital pillbox) in Uganda; neither has any financial interest in this technology. $\mathrm{JH}$ also consults for Merck and is also conducting research involving the use of evriMED1000 (a digital pillbox); she has no financial interest in this technology. $\mathrm{KHM}$ is providing mentorship for research evaluating the use of ingestible sensors; he does not have any financial interest in this technology.

Patient consent Not required.

Provenance and peer review Not commissioned; externally peer reviewed.

Data sharing statement This is a review article in which all studies described are available as published manuscripts or conference abstracts.

Open access This is an open access article distributed in accordance with the Creative Commons Attribution 4.0 Unported (CC BY 4.0) license, which permits others to copy, redistribute, remix, transform and build upon this work for any purpose, provided the original work is properly cited, a link to the licence is given, and indication of whether changes were made. See: http://creativecommons.org/ licenses/by/4.0

\section{REFERENCES}

1. World Health Organization. Global tuberculosis report. Geneva: WHO, 2015.

2. Thomas A, Gopi PG, Santha T, et al. Predictors of relapse among pulmonary tuberculosis patients treated in a DOTS programme in South India. Int J Tuberc Lung Dis 2005;9:556-61.

3. Vijay S, Kumar P, Chauhan LS, et al. Risk factors associated with default among new smear positive TB patients treated under DOTS in India. PLoS One 2010;5:e10043.

4. Thomas B, Watson B, Senthil EK, et al. Alcohol intervention strategy among tuberculosis patients: a pilot study from South India. Int J Tuberc Lung Dis 2017;21:947-52.

5. Thomas BE, Shanmugam P, Malaisamy M, et al. Psycho-socioeconomic issues challenging multidrug resistant tuberculosis patients: a systematic review. PLoS One 2016;11:e0147397.

6. Craig GM, Daftary A, Engel N, et al. Tuberculosis stigma as a social determinant of health: a systematic mapping review of research in low incidence countries. Int J Infect Dis 2017;56:90-100.

7. Munro SA, Lewin SA, Smith HJ, et al. Patient adherence to tuberculosis treatment: a systematic review of qualitative research. PLoS Med 2007;4:e238.

8. Shubber Z, Mills EJ, Nachega JB, et al. Patient-reported barriers to adherence to antiretroviral therapy: a systematic review and metaanalysis. PLoS Med 2016;13:e1002183.

9. Sagbakken M, Frich JC, Bjune GA, et al. Ethical aspects of directly observed treatment for tuberculosis: a cross-cultural comparison. BMC Med Ethics 2013;14:25.

10. Karumbi J, Garner P. Directly observed therapy for treating tuberculosis. Cochrane Database Syst Rev 2015;5:Cd003343.

11. Pasipanodya JG, Gumbo T. A meta-analysis of self-administered vs directly observed therapy effect on microbiologic failure, relapse, and acquired drug resistance in tuberculosis patients. Clin Infect Dis 2013;57:21-31.

12. Tian JH, Lu ZX, Bachmann MO, et al. Effectiveness of directly observed treatment of tuberculosis: a systematic review of controlled studies. Int J Tuberc Lung Dis 2014;18:1092-8.

13. Pew Research Center. Cell phones in Africa. Communication Lifeline, 2015

14. Pop-Eleches $\mathrm{C}$, Thirumurthy $\mathrm{H}$, Habyarimana JP, et al. Mobile phone technologies improve adherence to antiretroviral treatment in a resource-limited setting: a randomized controlled trial of text message reminders. AIDS 2011;25:825-34.

15. Haberer JE, Musiimenta A, Atukunda EC, et al. Short message service (SMS) reminders and real-time adherence monitoring improve antiretroviral therapy adherence in rural Uganda. AIDS 2016;30:1295-9.

16. Haberer JE, Musinguzi N, Tsai AC, et al. Real-time electronic adherence monitoring plus follow-up improves adherence 
compared with standard electronic adherence monitoring. AIDS 2017;31:169-71.

17. Sabin LL, Bachman DeSilva M, Gill CJ, et al. Improving adherence to antiretroviral therapy with triggered real-time text message reminders: the China adherence through technology study. J Acquir Immune Defic Syndr 2015;69:551-9.

18. Vervloet M, van Dijk L, de Bakker DH, et al. Short- and long-term effects of real-time medication monitoring with short message service (SMS) reminders for missed doses on the refill adherence of people with type 2 diabetes: evidence from a randomized controlled trial. Diabet Med 2014;31:821-8.

19. Checchi KD, Huybrechts KF, Avorn J, et al. Electronic medication packaging devices and medication adherence: a systematic review. JAMA 2014;312:1237-47.

20. Demonceau J, Ruppar T, Kristanto P, et al. Identification and assessment of adherence-enhancing interventions in studies assessing medication adherence through electronically compiled drug dosing histories: a systematic literature review and metaanalysis. Drugs 2013;73:545-62.

21. de Bruin M, Oberjé EJM, Viechtbauer W, et al. Effectiveness and cost-effectiveness of a nurse-delivered intervention to improve adherence to treatment for HIV: a pragmatic, multicentre, open-label, randomised clinical trial. Lancet Infect Dis 2017:17:595-604.

22. Obermeyer Z, Abbott-Klafter J, Murray CJ. Has the DOTS strategy improved case finding or treatment success? An empirical assessment. PLoS One 2008:3:e1721.

23. World Health Organization (WHO). Handbook for the use of digital technologies to support tuberculosis medication dherence. Geneva: WHO, 2017.

24. Ngwatu BK, Nsengiyumva NP, Oxlade O, et al. The impact of digital health technologies on tuberculosis treatment: a systematic review. Eur Respir J 2018;51:1701596.

25. Bayer R, Wilkinson D, Bayer R. Directly observed therapy for tuberculosis: history of an idea. Lancet 1995;345:1545-8.

26. Yellappa V, Lefèvre P, Battaglioli T, et al. Coping with tuberculosis and directly observed treatment: a qualitative study among patients from South India. BMC Health Serv Res 2016;16:283.

27. Wynne A, Richter S, Banura L, et al. Challenges in tuberculosis care in Western Uganda: health care worker and patient perspectives. International Journal of Africa Nursing Sciences 2014;1:6-10.

28. Sagbakken M, Frich JC, Bjune G. Barriers and enablers in the management of tuberculosis treatment in Addis Ababa, Ethiopia: a qualitative study. BMC Public Health 2008;8:11.

29. Lei X, Huang K, Liu Q, et al. Are tuberculosis patients adherent to prescribed treatments in China? Results of a prospective cohort study. Infect Dis Poverty 2016;5:38.

30. Hou WL, Song FJ, Zhang NX, et al. Implementation and community involvement in DOTS strategy: a systematic review of studies in China. Int J Tuberc Lung Dis 2012;16:1433-40.

31. Benbaba S, Isaakidis P, Das M, et al. Direct Observation (DO) for drug-resistant tuberculosis: do We really dO? PLoS One 2015;10:e0144936

32. Ware NC, Pisarski EE, Tam M, et al. The Meanings in the messages: how SMS reminders and real-time adherence monitoring improve antiretroviral therapy adherence in rural Uganda. AIDS 2016;30:1287-94.

33. Moulding TS. Viewpoint: adapting to new international tuberculosis treatment standards with medication monitors and DOT given selectively. Trop Med Int Health 2007;12:1302-8.

34. Cross A, Gupta N, Liu B. 99DOTS: a low-cost approach to monitoring and improving medication adherence. Tenth international conference on information and communication technologies and development (ICTD 2019), Ahmedabad, India, 2019.

35. AiCure, 2017. LA county department of public health: monitoring treatment to tuberculosis disease and latent tuberculosis infection. https://aicure.com/clinical_evidence/using-aicure-to-meausureand-support-tuberculosis-treatment-a-pilot-study/ (accessed 18 Oct 2017).

36. Labovitz DL, Shafner L, Reyes Gil M, et al. Using artificial intelligence to reduce the risk of nonadherence in patients on anticoagulation therapy. Stroke 2017;48:1416-9.

37. Belknap R, Weis S, Brookens A, et al. Feasibility of an ingestible sensor-based system for monitoring adherence to tuberculosis therapy. PLoS One 2013;8:e53373.

38. Browne S, Moser K, Low J. Wirelessly observed therapy is accurate and confirms more TB medication doses than directly observed therapy. Guadalajara, Mexico: 48th Union World Conference on Lung Health, 2017.
39. Liu X, Lewis JJ, Zhang $\mathrm{H}$, et al. Effectiveness of electronic reminders to improve medication adherence in tuberculosis patients: a cluster-randomised trial. PLoS Med 2015;12:e1001876.

40. van Gemert-Pijnen JE, Nijland N, van Limburg M, et al. A holistic framework to improve the uptake and impact of eHealth technologies. J Med Internet Res 2011;13:e111.

41. Yusof MM, Kuljis J, Papazafeiropoulou A, et al. An evaluation framework for health information systems: human, organization and technology-fit factors (HOT-fit). Int J Med Inform 2008;77:386-98.

42. Venkatesh V, Morris M, Davis GB, et al. User acceptance of information technology: toward a unified view. MIS Quarterly 2003:27:425-78.

43. Holden RJ, Karsh BT. The technology acceptance model: its past and its future in health care. J Biomed Inform 2010;43:159-72.

44. Campbell JI, Aturinda I, Mwesigwa E, et al. The Technology Acceptance Model for Resource-Limited Settings (TAM-RLS): a novel framework for mobile health interventions targeted to low-literacy end-users in resource-limited settings. AIDS Behav 2017;21:3129-40.

45. DiStefano MJ, Schmidt $\mathrm{H}$. mHealth for tuberculosis treatment adherence: a framework to guide ethical planning, implementation, and evaluation. Global Health: Science and Practice 2016;4:211-21.

46. Campbell Jl, Eyal N, Musiimenta A, et al. Ethical questions in medical electronic adherence monitoring. J Gen Intern Med 2016;31:338-42.

47. Sharp RR. Ingestible drug adherence monitors: trending toward a surveillance society? Am J Bioeth 2015;15:1-2.

48. Katz IT, Ryu AE, Onuegbu AG, et al. Impact of HIV-related stigma on treatment adherence: systematic review and meta-synthesis. $J$ Int AIDS Soc 2013;16:18640.

49. bridges.org. Evaluation of the on cue compliance service pilot. South Africa Cape Town: Testing the use of SMS reminders in the treatment of Tuberculosis in Cape Town, 2005.

50. Siedner MJ, Haberer JE, Bwana MB, et al. High acceptability for cell phone text messages to improve communication of laboratory results with HIV-infected patients in rural Uganda: a cross-sectional survey study. BMC Med Inform Decis Mak 2012;12:56.

51. Albino S, Tabb KM, Requena D, et al. Perceptions and acceptability of short message services technology to improve treatment adherence amongst tuberculosis patients in Peru: a Focus Group Study. PLoS One 2014;9:e95770.

52. Iribarren S, Beck S, Pearce PF, et al. TextTB: a mixed method pilot study evaluating acceptance, feasibility, and exploring initial efficacy of a text messaging intervention to support TB treatment adherence. Tuberc Res Treat 2013;2013:349394.

53. Garfein RS, Collins K, Muñoz F, et al. Feasibility of tuberculosis treatment monitoring by video directly observed therapy: a binational pilot study. Int J Tuberc Lung Dis 2015;19:1057-64.

54. Hoffman JA, Cunningham JR, Suleh AJ, et al. Mobile direct observation treatment for tuberculosis patients: a technical feasibility pilot using mobile phones in Nairobi, Kenya. Am J Prev Med 2010;39:78-80.

55. Mirsaeidi M, Farshidpour M, Banks-Tripp D, et al. Video directly observed therapy for treatment of tuberculosis is patient-oriented and cost-effective. Eur Respir J 2015;46:871-4.

56. Zúñiga ML, Collins $\mathrm{K}$, Muñoz F, et al. A qualitative study exploring stakeholder perceptions of video directly observed therapy for monitoring tuberculosis treatment in the US-Mexico border region. J Mob Technol Med 2016;5:12-23.

57. Liu X, Blaschke T, Thomas B, et al. Usability of a Medication Event Reminder Monitor System (MERM) by providers and patients to improve adherence in the management of tuberculosis. Int $J$ Environ Res Public Health 2017;14:1115.

58. Mohammed S, Siddiqi O, Ali O, et al. User engagement with and attitudes towards an interactive SMS reminder system for patients with tuberculosis. J Telemed Telecare 2012;18:404-8.

59. Wade VA, Karnon J, Eliott JA, et al. Home videophones improve direct observation in tuberculosis treatment: a mixed methods evaluation. PLoS One 2012;7:e50155.

60. Hermans SM, Elbireer S, Tibakabikoba $\mathrm{H}$, et al. Text messaging to decrease tuberculosis treatment attrition in TB-HIV coinfection in Uganda. Patient Prefer Adherence 2017:11:1479-87.

61. Siedner MJ, Lankowski A, Musinga D, et al. Optimizing network connectivity for mobile health technologies in sub-Saharan Africa. PLoS One 2012; 7:e45643-11.

62. Lei $X$, Liu $Q$, Wang $H$, et al. Is the short messaging service feasible to improve adherence to tuberculosis care? A cross-sectional study. Trans R Soc Trop Med Hyg 2013;107:666-8.

63. Saunders MJ, Wingfield T, Tovar MA, et al. Mobile phone interventions for tuberculosis should ensure access to mobile 
phones to enhance equity - a prospective, observational cohor study in Peruvian shantytowns. Trop Med Int Health 2018;23:850-9.

64. Story A, Garfein RS, Hayward A, et al. Monitoring therapy compliance of tuberculosis patients by using video-enabled electronic devices. Emerg Infect Dis 2016;22:538-40.

65. Gassanov MA, Feldman LJ, Sebastian A, et al. The use of videophone for directly observed therapy for the treatment of tuberculosis. Can J Public Health 2013;104:272.

66. Sinkou H, Hurevich $\mathrm{H}$, Rusovich V, et al. Video-observed treatment for tuberculosis patients in Belarus: findings from the first programmatic experience. Eur Respir J 2017;49:1602049.

67. Chuck C, Robinson E, Macaraig M, et al. Enhancing management of tuberculosis treatment with video directly observed therapy in New York City. Int J Tuberc Lung Dis 2016;20:588-93.

68. Mohammed S, Glennerster R, Khan AJ. Impact of a daily SMS medication reminder system on tuberculosis treatment outcomes: a randomized controlled trial. PLoS One 2016;11:e0162944.

69. Deschamps AE, Van Wijngaerden E, Denhaerynck K, et al. Use of electronic monitoring induces a 40-day intervention effect in HIV patients. J Acquir Immune Defic Syndr 2006;43:247-8.

70. Vrijens B, De Geest S, Hughes DA, et al. A new taxonomy for describing and defining adherence to medications. Br J Clin Pharmacol 2012;73:691-705.

71. Elizaga J, Friedland JS. Monitoring compliance with antituberculous treatment by detection of isoniazid in urine. Lancet 1997;350:1225-6.

72. Soobratty MR, Whitfield R, Subramaniam K, et al. Point-of-care urine test for assessing adherence to isoniazid treatment for tuberculosis. Eur Respir J 2014;43:1519-22.

73. Whitfield R, Cope GF. Point-of-care test to monitor adherence to anti-tuberculous treatment. Ann Clin Biochem 2004:41:411-3.

74. Bangsberg DR, Hecht FM, Charlebois ED, et al. Comparing objective measures of adherence to HIV Antiretroviral therapy: electronic medication monitors and unannounced pill counts. AIDS Behav 2001;5:275-81.

75. Huan S, Chen R, Liu X. Operational feasibility of medication monitors in monitoring treatment adherence among TB patients. Chin J Antituberculosis 2012;34:419-24.

76. Bionghi N, Daftary A, Maharaj B, et al. Pilot evaluation of a secondgeneration electronic pill box for adherence to Bedaquiline and antiretroviral therapy in drug-resistant TB/HIV co-infected patients in KwaZulu-Natal, South Africa. BMC Infect Dis 2018;18:171.

77. Pai M, Schumacher SG, Abimbola S. Surrogate endpoints in global health research: still searching for killer apps and silver bullets? BMJ Glob Health 2018;3:e000755.

78. Bediang G, Stoll B, Elia N, et al. SMS reminders to improve the tuberculosis cure rate in developing countries (TB-SMS Cameroon): a protocol of a randomised control study. Trials 2014;15:35.

79. Yoeli E, Rathauser J, Rand D. Self-verification and behavioral interventions via mobile phones drastically improves tuberculosis treatment success in a randomized control trial OA-136-12. Guadalajara, Mexico: 48th Union World Conference on Lung Health, 2017.

80. Broomhead S, Mars M. Retrospective return on investment analysis of an electronic treatment adherence device piloted in the Northern Cape Province. Telemed J E Health 2012;18:24-31.

81. Ying R, Sharma M, Heffron R, et al. Cost-effectiveness of preexposure prophylaxis targeted to high-risk serodiscordant couples as a bridge to sustained ART use in Kampala, Uganda. J Int AIDS Soc 2015;18(4 Suppl 3):20013.

82. Phillips AN, Cambiano V, Nakagawa F, et al. Cost effectiveness of potential ART adherence monitoring interventions in Sub-Saharan Africa. PLoS One 2016;11:e0167654.

83. Krueger K, Ruby D, Cooley P, et al. Videophone utilization as an alternative to directly observed therapy for tuberculosis. Int $J$ Tuberc Lung Dis 2010;14:779-81.

84. Achmat Z. Science and social justice: the lessons of HIV/AIDS activism in the struggle to eradicate tuberculosis. Int $J$ Tuberc Lung Dis 2006;10:1312-7.
85. Sweetland AC, Kritski A, Oquendo MA, et al. Addressing the tuberculosis-depression syndemic to end the tuberculosis epidemic. Int J Tuberc Lung Dis 2017;21:852-61.

86. Ambaw F, Mayston R, Hanlon C, et al. Untreated depression and tuberculosis treatment outcomes, quality of life and disability, Ethiopia. Bull World Health Organ 2018;96:243-55.

87. Cross A, Kumar M, Soren P. 99DOTS: monitoring and improving TB medication adherence using mobile phones and augmented packaging OA-414-05. Cape Town, South Africa: Union World Conference on Lung Health, 2015.

88. Subbaraman R, Daftary A. Monitoring and improving adherence to tuberculosis medications. In: Pai M, ed. Lets talk TB. 3rd edn. Mumbai, India: GP Clinics, 2017: 7-15.

89. Wang L, Zhang H, Ruan Y, et al. Tuberculosis prevalence in China, 1990-2010; a longitudinal analysis of national survey data. The Lancet 2014;383:2057-64.

90. Oberoi S, Gupta K V, Neha C. 99 DOTS Mini-Review. Int J Contemp Med Res 2016;3:2760-2.

91. Nhavoto JA, Grönlund Å, Chaquilla WP. SMSaúde: design, development, and implementation of a remote/mobile patient management system to improve retention in care for HIV/AIDS and tuberculosis patients. JMIR Mhealth Uhealth 2015;3:e26.

92. Nhavoto JA, Grönlund $\AA$, Klein GO. Mobile health treatment support intervention for HIV and tuberculosis in Mozambique: perspectives of patients and healthcare workers. PLoS One 2017;12:e0176051.

93. Hirsch-Moverman YDA, Yuengling KA, Saito S, et al. Using mHealth for HIV/TB treatment support in Lesotho: enhancing patientprovider communication in the START study. J Acquir Immune Defic Syndr 2017;1:S37-S43.

94. Howard AA, Hirsch-Moverman Y, Frederix K, et al. The start study to evaluate the effectiveness of a combination intervention package to enhance antiretroviral therapy uptake and retention during TB treatment among TB/HIV patients in Lesotho: rationale and design of a mixed-methods, cluster-randomized trial. Glob Health Action 2016;9:31543.

95. Fang XH, Guan SY, Tang L, et al. Effect of short message service on management of pulmonary tuberculosis patients in Anhui Province, China: a prospective, randomized, controlled study. Med Sci Monit 2017;23:2465-9.

96. Kumboyono K. Short message service as an alternative in the drug consumption evaluation of persons with tuberculosis in Malang, Indonesia. Jpn J Nurs Sci 2017;14:112-6.

97. Kunawararak P, Pongpanich S, Chantawong S, et al. Tuberculosis treatment with mobile-phone medication reminders in northern Thailand. Southeast Asian J Trop Med Public Health 2011;42:1444-51.

98. Nglazi MD, Bekker LG, Wood R, et al. Mobile phone text messaging for promoting adherence to anti-tuberculosis treatment: a systematic review. BMC Infect Dis 2013;13:566.

99. Macaraig M, Lobato MN, McGinnis Pilote K, et al. A national survey on the use of electronic directly observed therapy for treatment of tuberculosis. Journal of Public Health Management and Practice 2017:1.

100. Molton JS, Pang Y, Wang Z, et al. Prospective single-arm interventional pilot study to assess a smartphone-based system for measuring and supporting adherence to medication. BMJ Open 2016;6:e014194.

101. Nguyen TA, Pham MT, Nguyen TL, et al. Video directly observed therapy to support adherence with treatment for tuberculosis in Vietnam: a prospective cohort study. Int J Infect Dis 2017;65:85-9.

102. Musiimenta A. Real time tuberculosis medication adherence intervention in rural southwestern Uganda. Uganda: Fogarty International Center, National Institutes of Health, 2017.

103. de Sumari-de Boer IM, van den Boogaard J, Ngowi KM, et al. Feasibility of real time medication monitoring among HIV infected and TB patients in a resource-limited setting. AIDS Behav 2016;20:1097-107. 